\title{
Concentric manipulation and monitoring of protein-loaded superparamagnetic cargo using magnetophoretic spider web
}

\author{
Byeonghwa Lim ${ }^{1}$, Sri Ramulu Torati ${ }^{1}$, Kun Woo Kim, Xinghao Hu, Venu Reddy and CheolGi Kim
}

\begin{abstract}
A lab-on-a-chip (LOC) magnetophoretic system for the remotely controllable transport of magnetic particles actuated by thin permalloy magnetic tracks has been developed as a novel architecture composed of radii and spiral tracks resembling a spider web network, where the network tracks have the asymmetric and anisotropic magnetic properties for the directional transportation of particles (cargos). A planar Hall resistance (PHR) sensor is integrated with the web networks, and the manipulation and detection are achieved via superparamagnetic particles with dual functions as a biomolecule cargo for transportation and labels for monitoring. The streptavidin protein-coated magnetic particles are precisely manipulated toward the PHR sensor surface via the radii and spiral tracks by applying an external rotating magnetic field. The stray field was analyzed in terms of the particle coverage on the sensor surface, where the sensor signal linearly varies with the number of particles on the sensor surface. This allows the effective collection of low-density biomolecule carriers to one specific point and monitors the accumulated carriers. The developed novel technology could affect multiple fields, including bioassays, cell manipulation and separation and biomechanics.
\end{abstract}

NPG Asia Materials (2017) 9, e369; doi:10.1038/am.2017.37; published online 31 March 2017

\section{INTRODUCTION}

Significant developments have been made in biosensors integrated with lab-on-a-chip (LOC) platforms driven by the advances in biotechnology, nano/micro-technologies and microfluidics. ${ }^{1-6}$ These miniaturized, highly integrated systems have the potential to achieve high-resolution molecular detection, more rapid and multiplexed analyses and reduced reagent sample volume (that is, the $\mu \mathrm{l}$ or even pl range). ${ }^{2,7}$ Although innovative and more advanced sensors have been developed for specific biomolecule detection down to approximately $\mathrm{fM}$ resolution, ${ }^{8-12}$ the $\mathrm{nm}-\mu \mathrm{m}$-sized sensor resolution is not limited by the signal transduction limitation but instead by the lack of appropriate analyte transport in solution that governs the detection time and results in poor sensor performance.,13-15 Therefore, the control over transporting specific biomolecules to the sensing site is a prerequisite for an ideal LOC platform able to overcome diffusive accumulation in solution and achieve high-resolution detection of analytes and short detection times.

Since the late 1990s, magnetoresistive (MR) sensors have been used for sensing biomolecules via superparamagnetic nano/micro-particle labels ${ }^{11,12,16,17}$ that also act as bioanalyte carriers under applied magnetic field. Magnetic particles are stable and require minimal sample purification when they are used as labels in MR detection systems because of the negligible magnetic background of complex bioanalytes. ${ }^{14}$ Moreover, these MR sensors are compatible with established semiconductor processing technology and are very sensitive, inexpensive and easily integrated into LOC systems for miniaturization. ${ }^{18}$ Particularly, the high signal-to-noise ratio, small off-set voltage and linear response at small field of planar Hall resistance (PHR) sensors relative to other MR sensors make them promising candidates for the LOC-based biosensors for high-resolution detection. ${ }^{19}$

Recently, bioanalyte (for example, cells, DNA and proteins) transportation using superparamagnetic nano/micro-particle carriers have gained significant importance because of its potential application in molecular diagnosis and separation technology. ${ }^{20-22}$ Biomolecules can be easily conjugated to magnetic particles using their surface chemistry and then manipulated to the desired location. Various approaches to controlled magnetic transport systems have been reported. For example, an array of separated magnetic disks was successfully used to transport superparamagnetic particles through periodic patterns by changing the magnetic field direction. ${ }^{23-25}$ In these symmetric array patterns, the driving force underlying translocation is caused by the out-of-plane rotation of the magnetic field, and the particles move linearly along the plane of the rotating field by hopping through the periodic patterns. Although the particle moving direction can be controlled by the direction of the rotating

Department of Emerging Materials Science, DGIST, Daegu, Republic of Korea

${ }^{1}$ These authors contributed equally to this work.

Correspondence: Professor CG Kim, Department of Emerging Materials Science, DGIST, Techno Jungang-Daero, Hyeonpung-Myeon, Dalseong-Gun, Daegu 42988, Republic of Korea.

E-mail: cgkim@dgist.ac.kr

Received 10 September 2016; revised 24 December 2016; accepted 24 January 2017 
field plane in a two-dimensional platform, the particle positions cannot be controlled accurately.

Asymmetric shapes along the magnetic pathway patterns cause differences in the magnetostatic potential energy when the local field is parallel to the external field. Thus, the magnetic particle trajectory can be precisely controlled by shifting the energy minima across the substrate. ${ }^{26-28}$ Similarly, the lateral asymmetry pathway causes lateral differences in the field distribution, where particles move through ellipses and continuous edges of sawtooth, ${ }^{29,30}$ and magnetic conductor patterns, ${ }^{21,31-33}$ directionally controlled by the rotating field directions. Although the massive linear transportation of biofunctionalized particles was achieved by parallel pathways in our previous report, ${ }^{26}$ there has yet to be more precise transport of particles to a concentric point, and also there is still no magnetic architecture available for controlling low-density biocarriers as well as overcoming the low diffusion transport of biomolecules toward the sensing region.

In the present paper, to overcome the drawbacks of effective collection of biomolecule carriers by current available techniques, we established novel magnetic pattern architecture that resembled a spider web network. Specifically, the assemblies of several half-disk magnetic patterns were arranged as radii and spiral tracks, and the MR sensor was integrated at the center node of the spider web. This innovative magnetic spider web can achieve high concentric translocation of streptavidin-coated magnetic particles to one point and then diverge away from that point upon the application of a rotating magnetic field in clockwise and counterclockwise directions, respectively. Our efforts allowed collecting the streptavidin-coated carrier particles at far distances from the sensing area and monitoring them based on the integrated MR sensor. Here, the superparamagnetic particles not only play the role of biomolecular cargo for transportation, but also act as labels for the sensor, where the stray field was analyzed in terms of the particle coverage on the sensor surface. The developed novel platform substantially increases the collection capability of biomolecules even in low density and, thus, the sensitivity of the biosensor.

\section{MATERIALS AND METHODS}

\section{Materials}

The streptavidin-coated superparamagnetic particles with a diameter of $2.8 \mu \mathrm{m}$ (Dynabeads M-280) were purchased from Invitrogen (Waltham, MA, USA). The Wafermart Company (Cheongju, Korea) provided the silicon wafers coated with $200 \mathrm{~nm}$ thick $\mathrm{SiO}_{2}$. The photoresist (AZ 5214-E) and corresponding developer were purchased from AZ Electronic Materials (Seoul, South Korea). Poly(dimethylsiloxane) (Sylgard 184) was procured from Dowhitech Silicone Co. Ltd (Goyang, Korea). We used Atto-520 biotin from Sigma-Aldrich (St Louis, MO, USA) and phosphate buffer was obtained from Bioneer Company (Daejeon, Korea).

\section{Fabrication of the MR sensor on magnetic patterns}

The details of the MR sensor fabrication process have been described elsewhere. ${ }^{34}$ Briefly, the sensor was fabricated on a silicon wafer using a standard photolithography technique. The sensor was composed of a $\mathrm{Ta}(5) / \mathrm{NiFe}(10) / \mathrm{IrMn}(10) / \mathrm{Ta}(5)(\mathrm{nm})$ film stack coated with $100 \mathrm{~nm}$ thick $\mathrm{SiO}_{2}$ and patterned into a PHR sensor electrode geometry. A uniform $7.96 \mathrm{kA} \mathrm{m}^{-1}$ magnetic field was applied parallel to the film plane during the sputtering process to induce unidirectional anisotropy of the NiFe layer via exchange coupling with the antiferromagnetic IrMn layer.

\section{Magnetic spider web design}

A conventional photolithography and lift-off technique was used to fabricate soft permalloy $\left(\mathrm{Ni}_{80} \mathrm{Fe}_{20}\right)$ patterns of $100 \mathrm{~nm}$ thickness on the passivated PHR sensor. The linear radii tracks consisted of a periodic array of half-disks with $5 \mu \mathrm{m}$ radii connected by narrow magnetic segments with lengths of $2 \mu \mathrm{m}$. Each radii track was also connected to several spiral tracks at a distance of $14 \mu \mathrm{m}$. Finally, the spiral track-integrated radii were inclined at an angle of $22.5^{\circ}$, similar shape to a spider web. We intentionally introduced the half-disk asymmetry in this design to confine the particles to the curved (upper) section of the magnetic track where the field gradients are strongest. The short magnetic segments connecting the half-disks were designed to impose an energy penalty for a particle to cross over the spiral track.

\section{Rotating magnetic field setup}

The driving force for the magnetic particle was achieved using a rotational magnetic field produced by passing current through pairs of solenoid coils with ferrite cores controlled by LabVIEW (National Instruments, Seoul, South Korea). ${ }^{26}$ The sense of the field rotation was adjusted by applying a phase difference of $\pm 90^{\circ}$ between the orthogonal coils with field magnitudes ranging from 0 to $15.9 \mathrm{kA} \mathrm{m}^{-1}$. The motion of the superparamagnetic particles on the pattern was tracked via video microscopy using an IMC-1040FT camera (IMI Tech, Anyang, South Korea) connected to a computer. The total experimental setup, including the rotating magnetic field device, and picture of the magnetic spider web chip is shown in Supplementary Figure S1.

\section{$\boldsymbol{\mu}$-MOKE measurement analysis}

The spatial magnetic state of the half-disk pattern was examined by micro-magneto optical Kerr effect ( $\mu$-MOKE) microscopy by applying an external magnetic field, where the sample was staged in between two electromagnets and the beam is focused to $\sim 3 \mu \mathrm{m}$ diameter spot on the sample surface. The Kerr signal variation is analyzed through analyzer and the images were observed through CCD camera. The angle of the magnetic field to sample varied by mounting the sample with different angles.

\section{Conjugation of Atto-520 biotin on streptavidin-coated particles}

Initially, $5 \mu \mathrm{l}$ of streptavidin-coated magnetic particles was washed several times with phosphate-buffered saline ( $\mathrm{pH}$ 7.4) to remove preservatives. Then, the magnetic particles were suspended in $90 \mu \mathrm{l}$ of phosphate-buffered saline. To this, $5 \mu \mathrm{l}$ of diluted Atto-520 ( $1 \mathrm{mg}$ of Atto-520 biotin per $200 \mu \mathrm{l}$ of ethanol) was added, and the solution was continuously stirred for $2-3 \mathrm{~h}$ at room temperature to successfully complete the streptavidin-biotin conjugation. Then, the particles were washed with phosphate-buffered saline several times to remove excess biotin.

\section{Finite element method for magnetic simulation}

The Maxwell 3D software (version 16.1, Ansys, Canonsburg, PA, USA) was utilized for magnetic simulation using the finite element method. Here, the measured magnetization hysteresis curve of a thin permalloy film with a thickness of $100 \mathrm{~nm}$ was used to model the magnetic field distribution and magnetostatic potential energy, as shown in Supplementary Figure S2.

The magnetic potential energy on a superparamagnetic particle around the half-disk pattern pathways is proportional to the square of local magnetic field strength at particle position. The potential energy acting on the particle is given as follows:

$$
U=-\frac{1 V \chi_{v}}{2 \mu_{\mathrm{o}}} \vec{B}^{2}, \quad \vec{B}=\mu_{\mathrm{o}}\left(\vec{H}_{\text {app }}+\vec{H}_{\text {pattern }}\right)
$$

where $V$ is the particle volume, $\chi_{\mathrm{v}}$ is the magnetic volume susceptibility of the particle (the magnetization of the surrounding medium is neglected), $\mu_{0}$ is the vacuum permeability and effective field $\vec{B}$ is the sum of applied $\left(H_{\text {app }}\right)$ and generated $\left(H_{\text {pattern }}\right)$ magnetic field from pattern at the particle position. The potential energy of the particles was determined using equation (1), and is plotted as a function of the external field orientation.

\section{Characterization of magnetic tracks and superparamagnetic particles}

Figures 1a and $\mathrm{b}$ present schematics of the inclined half-disk alignments corresponding to the spider web radii tracks, where the particles move toward the converging site (red arrows) and diverge away from the converging site 
b

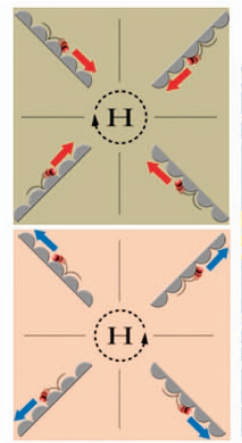

e

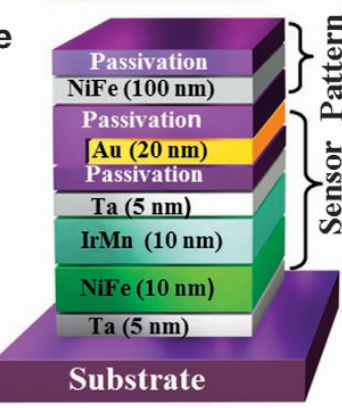

c
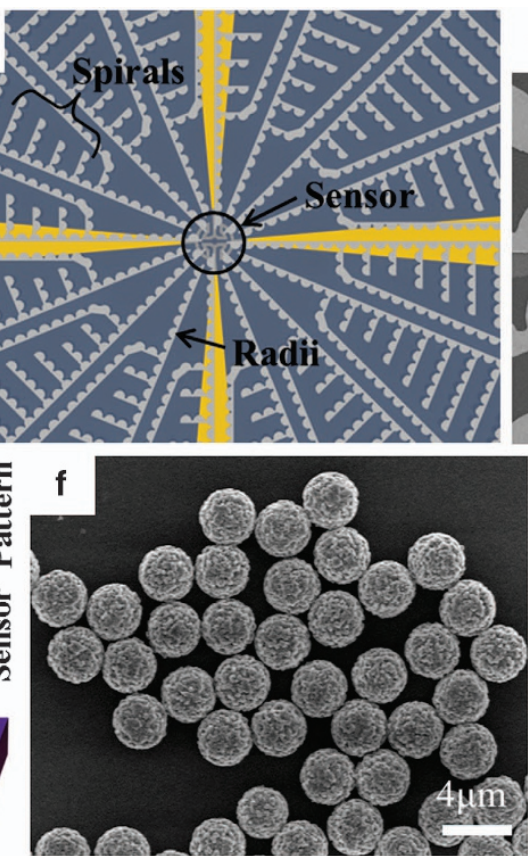
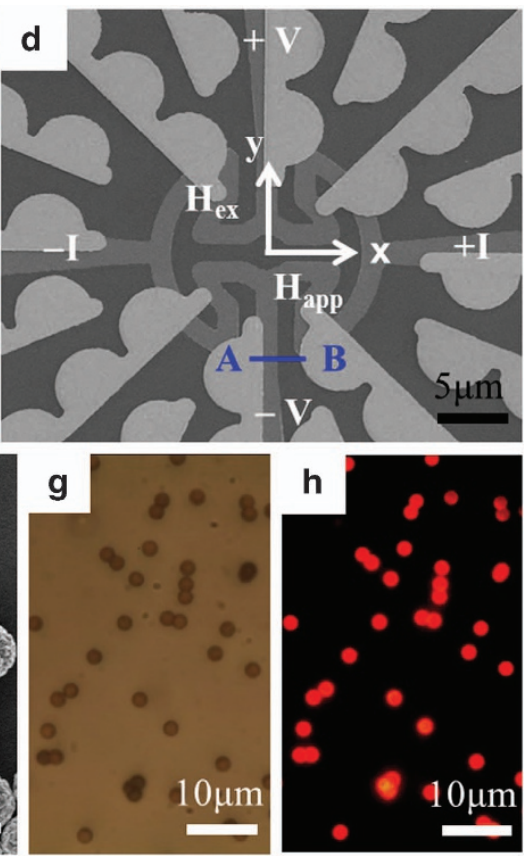

h

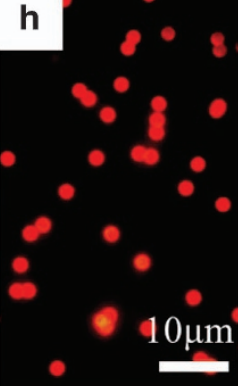

Figure 1 Conceptual design of magnetic spider web. (a) Schematics of the spider web radii with concentric movement of a magnetic particle under the clockwise rotation of the magnetic field. (b) The magnetic particle diverging under the counterclockwise rotation. (c) Schematic representation of the magnetoresistive (MR) sensor-integrated magnetic spider web using the NiFe magnetic pattern (thickness $100 \mathrm{~nm}$ ). (d) Scanning electron microscope (SEM) image of the MR (planar Hall resistance (PHR))-type sensor integrated with the spider web net and the measuring parameters. (e) Schematic cross-sectional view of the layered structures of the MR sensor and magnetic patterns, marked as A and B in Figure 1d. (f) SEM image of streptavidin-coated magnetic particles $(2.8 \mu \mathrm{m}$ diameter) used for manipulation and monitoring. (g) Bright-field and (h) fluorescence images of magnetic particles conjugated with Atto-520 biotin.

(blue arrows) when the applied magnetic field is rotated in clockwise and counterclockwise direction, respectively. The particles, which are actuated by the effective magnetic field, can linearly move from one point to another along these tracks. The linear radii track consisted of a half-disk pattern ( $5 \mu \mathrm{m}$ radius) connected by $2 \mu \mathrm{m}$ long narrow magnetic segments (Figure 1c). The angle between the two concentric radii tracks was $22.5^{\circ}$, and the distance between the two spiral tracks was $14 \mu \mathrm{m}$. We also arranged an assembly of $90^{\circ}$ joined linear tracks (T-junctions) to resemble a spider web spiral. The total size of the spider web network is nearly $2 \mathrm{~mm}$ in diameter. Finally, the MR sensor was integrated in the center of the spider web for the monitoring of biomolecules. The scanning electron microscope image of the MR sensor integrated at the center of the spider web patterns and the measurement parameters are shown in Figure 1d. The cross-sectional view of the material layers, electrode structure and passivation involved at the junction (shown as A and B in Figure 1d) is schematically shown in Figure 1e. The morphological structure of the streptavidin-coated magnetic particles with a $2.8 \mu \mathrm{m}$ diameter was observed using scanning electron microscope (Figure 1f). The streptavidin-coated particles were functionalized with Atto-520 biotin to display fluorescence. The confirmation of the Atto-520 biotin conjugation to the magnetic particles was confirmed by fluorescence microscopy. Figures $1 \mathrm{~g}$ and $\mathrm{h}$ represent the bright-field and fluorescence images, respectively, of the particles conjugated with Atto-520 biotin. These images clearly show that the magnetic particles exhibit highly intense fluorescence.

\section{RESULTS AND DISCUSSION}

The basic concept relies on the construction of multiple T-junction tracks to connect the radii and spirals of the magnetic spider web network. For this particular geometry, upon the clockwise rotation of the magnetic field, the carrier particles on the radii track move toward the centrally placed magnetic sensor by crossing the $\mathrm{T}$-junction because of the energy minima of the magnetic pattern toward the converging site (Figure 2a and Supplementary Video S1). In addition, the particles on the spiral track approach the radii track and then move toward the central region. A high-magnification image of the particle trajectories along the web network junction is shown in Figure $2 b$, in which the white arrows indicate the particle path, and the black dotted rectangle identifies the linear track around the junction. The fluorescence image of the particles on the concentric site of the magnetic spider web is shown in Figure 2c (Supplementary Video S2). The counterclockwise rotation of the magnetic field shifts the particles away from the spider web center (Figure 2d and Supplementary Video S1).

However, unlike clockwise rotation, when the magnetic field rotates in counterclockwise direction, the particles cannot cross the $\mathrm{T}$-junction because of the energy barrier and, thus, follow another outgoing curved pattern that is called diverging radii (Figure 2e; white arrows show the outgoing particle pathway, and the black dotted rectangle show the spider web $\mathrm{T}$-junction and diverging radii). Actually, this diverging radii track plays a dual role of converging and diverging but the most important function of these radii is diverging of the particles from center node. If the diverging radii track is not available, then the particle moves to the $\mathrm{T}$-junction where it cannot cross the junction and moves toward the edge of spiral and revolves around the half-disk of the edge of spiral. The fluorescence image of particles along the diverging radii track is shown in Figure $2 \mathrm{f}$ (Supplementary Video S2).

The particle movement along the linear track is based on the asymmetric feature of the local magnetic state of half-disk pattern. ${ }^{26}$ The spatial magnetic state of the half-disk pattern was examined by $\mu$-MOKE microscopy by applying an external magnetic field. Figure $3 \mathrm{a}$ shows the schematic drawing for $\mu$-MOKE measurement system with $3 \mu \mathrm{m}$ spatial resolution, where the relative magnetization 

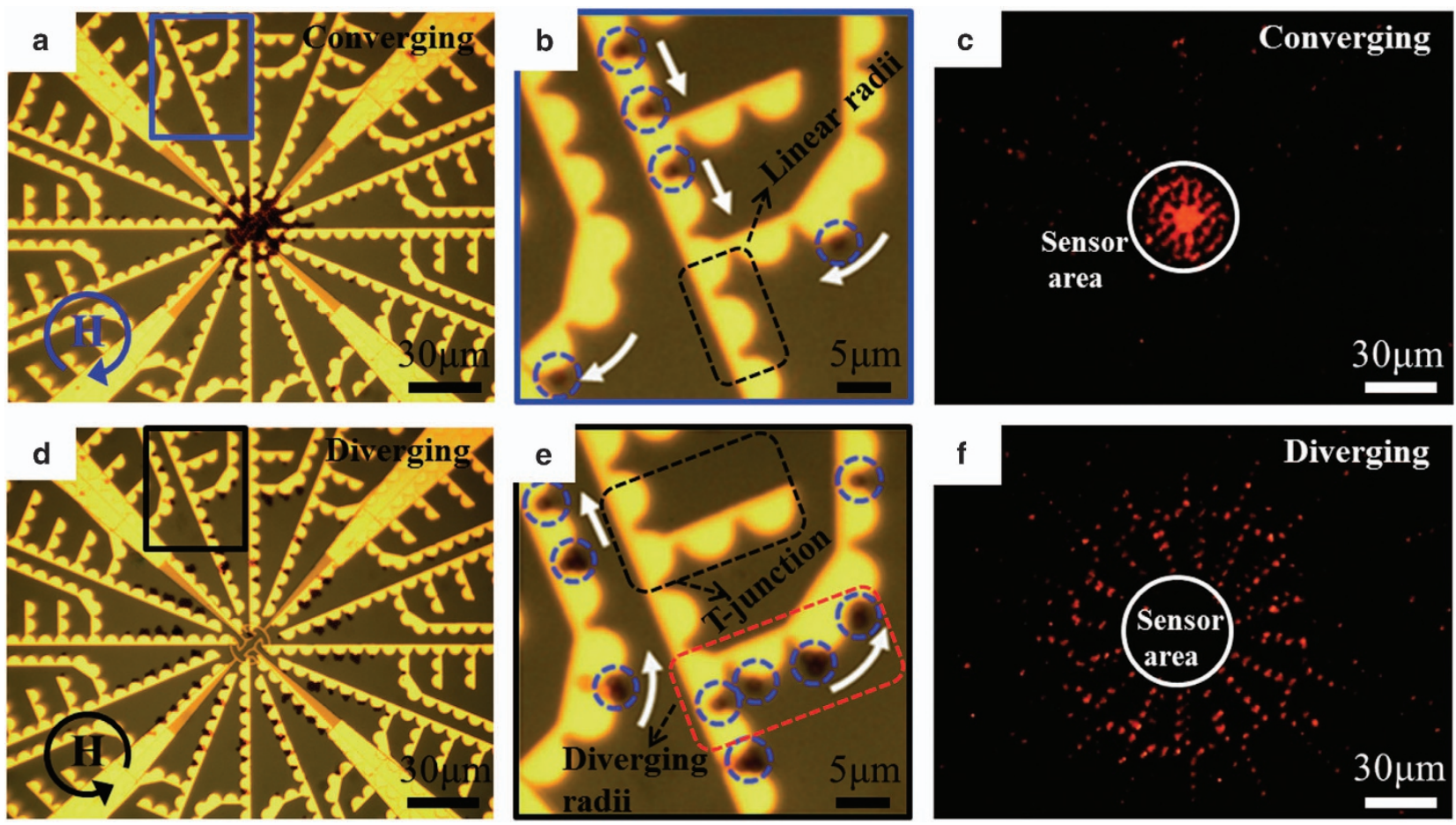

Figure 2 Controlled function of the magnetic spider web. (a) The particles drawn toward the center of the spider web under the clockwise magnetic field rotation. (b) The high-magnification image of particle trajectories along the web network junction (white arrows indicate the path of the particles, and the black dotted rectangle shows the linear radii track around the junction). (c) Fluorescence image of the particles at the concentric site of the magnetic spider web (the white circle indicates the sensor area). (d) The particles dispersed away from the spider web center under the counterclockwise magnetic field rotation. (e) The high-magnification image of the dispersion pathway of the magnetic particles (white arrows show the path of the dispersed particles, and the black dotted rectangle indicates the spider web T-junction where the magnetic particles cannot cross the T-junction and follow the curvy spiral track). (f) Fluorescence image of the particles at the diverging site of the spider web.

is related with the polarization angle change of reflected light from magnetized surface, that is Kerr signal. The longitudinal Kerr signal is proportional to the angle of local magnetization relative to the optical plane of incident light, where the skin depth is to be $\sim 30 \mathrm{~nm} .{ }^{35}$ As shown in Figure $3 \mathrm{~b}$, local magnetization loops measured at $0^{\circ}$ cyclic field over half-disk patterns are not uniform. As whole, these measurements mean that the magnetization variation is not uniform during the rotation of field, indicating that the anisotropic feature is accompanied near the pattern edge. Because the stray field is caused by local magnetization, magnetic energy variation is not uniform during the field rotation.

Here, the sample stage was rotated relative to a fixed incidence and reflected light direction for the measuring angle variation. As in Figure $3 c$, variation of relative magnetization, that is, the change of the angle between magnetization and plane of incident light, is drastically decreased for linear flat surface rather than coercivity. Comparing magnetization loops for flat and curved, P1 and P2, in Figure 3d, relative magnetization at $\mathrm{P} 1$ decreases with varied angle, but its variation is not so significant in P2, indicating the directional change of magnetization, where anisotropic feature is distinctive near P1.

Although there is anisotropic variation of local stray fields during the field rotation, the motion of the particles along the linear and T-junction tracks is more clearly examined by simulation of time-varying magnetic energy. ${ }^{26}$ The insert of Figure 4a represents the schematics of particle movement along the T-junction track, consisted of patterns 1 and 2. The minimum energy near the T-junction is slightly modified relative to the single pattern element and has two traces along patterns 1 and 2. As shown in Figure 4a, trace of minimum energy along the curve of pattern 1 is lower than that of pattern 2, even though positional change of minimum energy amount is to be $32.5 \%\left(-0.95 \times 10^{5}\right.$ to $\left.-1.38 \times 10^{5} k_{\mathrm{B}} T\right)$. It indicates that the particle could not move toward the flat edge of pattern 2 and the particle can easily cross the junction when the applied field rotates in clockwise rotation.

However, when the applied field rotates in counterclockwise direction, the particle moves upward at junction. This phenomenon can also be described in terms of the energy barrier formed between the patterns. Considering different arc positions along the curves of the patterns 1 and 2 (Figure $4 \mathrm{~b}$ ) from $70^{\circ}$ to $140^{\circ}$, the particle moves along pattern 1 at $90^{\circ}, 120^{\circ}$ and $140^{\circ}$ with minimum energy positions. The minimum energy of pattern 2 marked as red solid line is lower than that of pattern 1, marked as black dotted line, as shown in Figure $4 \mathrm{~b}$. Therefore, the particle moves toward the pattern 2, even though positional variation of minimum energy is nearly $12.2 \%\left(-1.22 \times 10^{5}\right.$ to $\left.-1.38 \times 10^{5} k_{\mathrm{B}} T\right)$ because the higher energy of pattern 1 at $90^{\circ}$ prevents the particle from moving toward pattern 1 . Thus, under counterclockwise rotation, the particles cannot cross the T-junction.

However, if the diverging radii track is not available, then the particles move toward the edge of track, pattern 3 from pattern 2, and rotate there across the half-disk element instead of moving on the flat edge of pattern 3 toward the flat edge of pattern 2, as schematically shown in inset of Figure 4c. If we consider different arc positions along the curves of the edge of pattern 3 (Figure 4c, inset shows schematic of pattern 3 ) from $180^{\circ}$ to $270^{\circ}$, the minimum energy of curved edge of half-disk marked as red line is lower than that of flat 
a
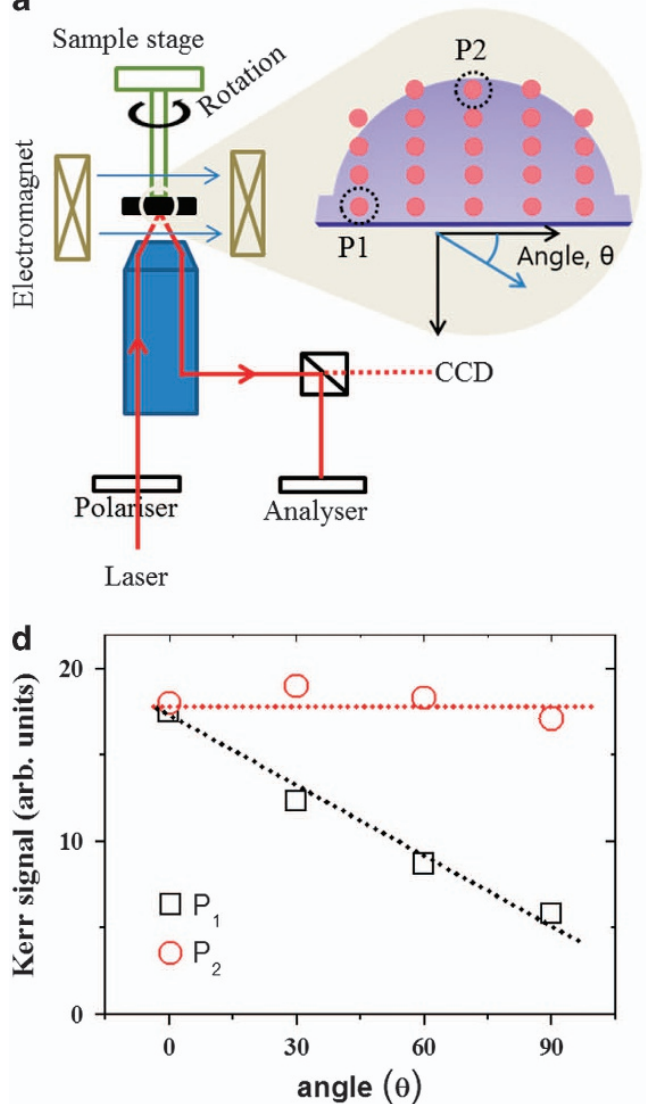

b

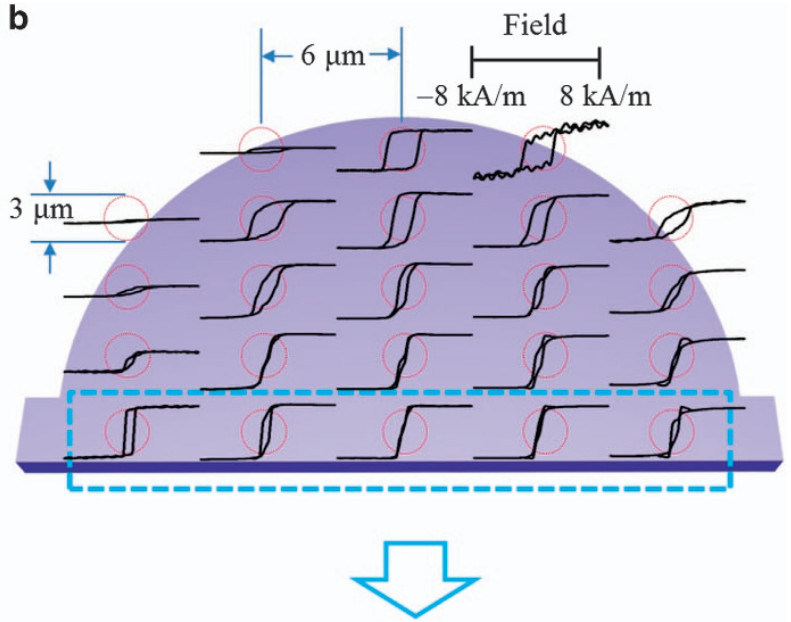

C

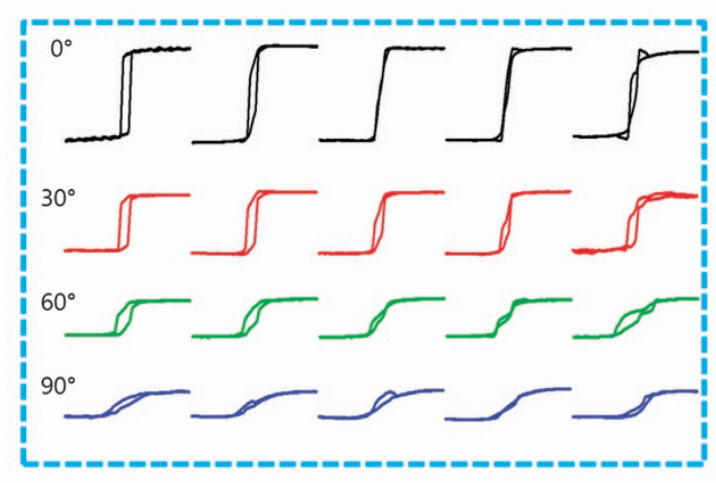

Figure 3 The micro-magneto optical Kerr effect ( $\mu$-MOKE) analysis of half-disk pattern in spider web. (a) Schematic drawing for $\mu$-MOKE measurement system. (b) The local distribution of longitudinal Kerr signal, at $\theta=0^{\circ}$, for entire surface of a half-disk pattern. (c) Angular variation of Kerr signal for flatedge points. (d) Relative variation of Kerr signal with different angles for flat edge (P1) and curved edge (P2).

edge, marked as dotted black line (Figure 4c). Hence, the particle moves to the curved edge of half-disk element of pattern 3 at the edge and just rotates there instead of moving anywhere (Figure $4 \mathrm{~d}$ ). Therefore, it is required to arrange diverging radii track where the particles shift their direction to the outgoing linear track (diverging radii), in agreement with the experimental results shown in the red rectangle in Figure 2e. In fact, the diverging radii track plays a dual role of both converging and diverging; however, its main function is the dispersing of particles from the center, where the converging radii can only act for particle collections.

In $1 \mathrm{~Hz}$ rotating field, nearly all particles within the reach of the spider web network were concentrated at the center point in $<1 \mathrm{~min}$. However, the focusing timescales can be significantly faster if higher rotation frequencies are used (for example, $10 \mathrm{~Hz}$ ). Spider web networks can cover large surfaces. Therefore, low-density particles can be uniformly controlled to move from an arbitrary initial point to a small central region at a uniform rate. In contrast, conventional magnetic separation/collection techniques are highly sensitive to the particle's initial positions. Thus, particles far from the magnetic collection apparatus represent the rate-limiting step in decreasing the collection time. In this demonstration, we achieved particle focusing with local concentrations several orders of magnitude higher than those in the bulk solution. Thus, high concentrations can be obtained even for low-density particles by expanding the reach of the spider web area. In addition, by optimizing the parameters such as magnetic pattern width and length, and the applied rotating field, ${ }^{36}$ the magnetic spider web can transport even anisotropic superparamagnetic nanochains or clusters if they do not change their shape or maintained fixed shape by applying the magnetic field during the process of manipulation. However, if the nanochains or cluster will change their shape during the manipulation, ${ }^{37}$ then the magnetic spider web could not manipulate nanoclusters precisely.

If the sensor is placed at the spider web center, then it would experience locally enhanced biomolecule sensing via the carriers, leading to better sensitivity and efficiency. Thus, we integrated the MR sensor at the center node on the spider web to monitor the presence of particles as a function of time. A picture of the integrated active MR sensor in the spider web junction and the dummy sensor (Supplementary Figure S3a for the image of the dummy sensor with measuring parameters) fabricated in the same batch are shown in Figure 5a. The MR sensor profiles for the active sensor in the spider web pattern and the dummy sensor are shown in Figure 5b. The active MR signal shows the hysteresis resulting from the field caused by the magnetic hysteretic effect of the patterns. However, no hysteresis is observed in the dummy MR sensor that is far distant from the spider web pattern elements. The calculated sensitivity of the sensor is $0.2 \mathrm{~V} \mathrm{mT}^{-1}$ near the field region of $\sim 10 \mathrm{mT}$, as depicted in Figure 5b.

The particle on the top surface of the MR sensor leads to stray fields around its periphery that opposes the external magnetic field, as schematically shown in Supplementary Figure S3b. The stray field of a single particle is given as the function of magnetic parameters of the particle and the distance from the sensor surface (Supplementary 

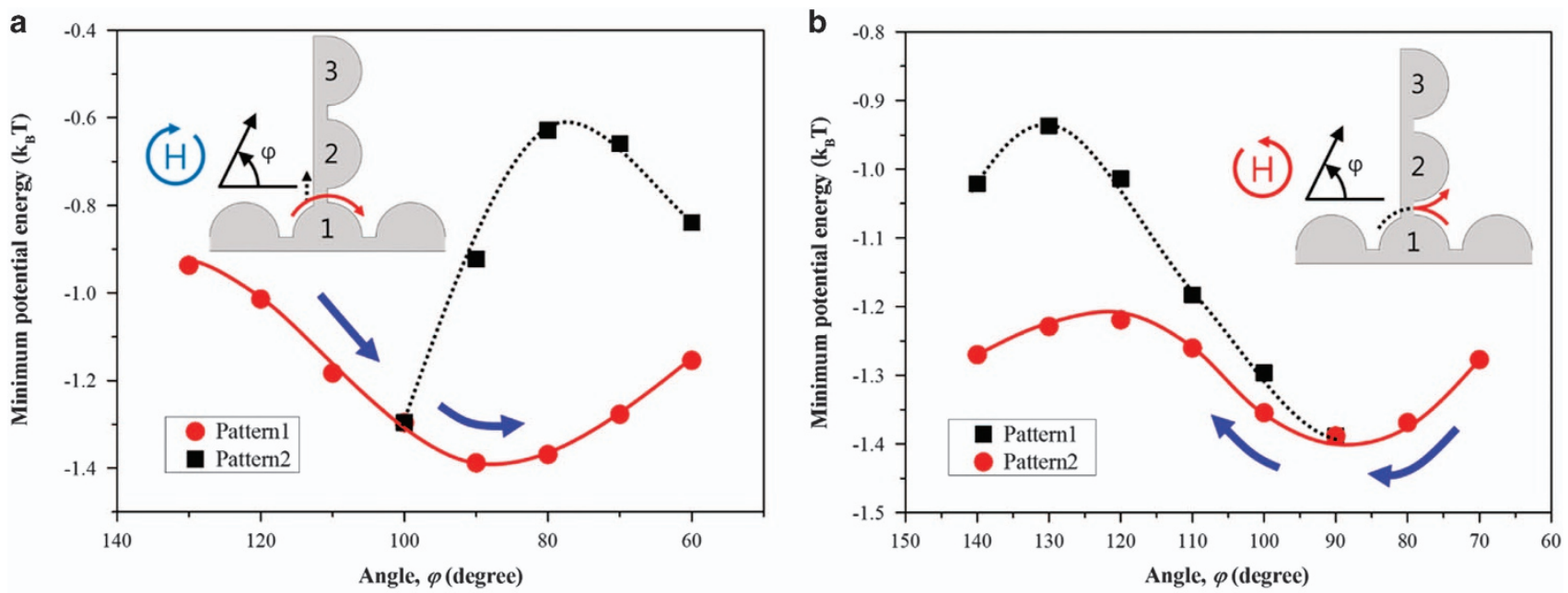

C

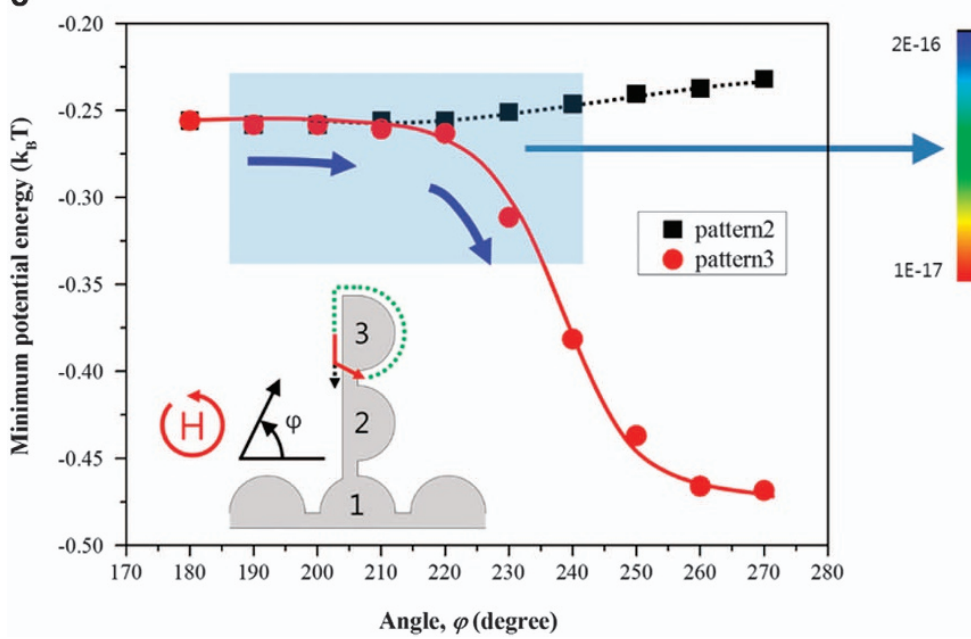

d
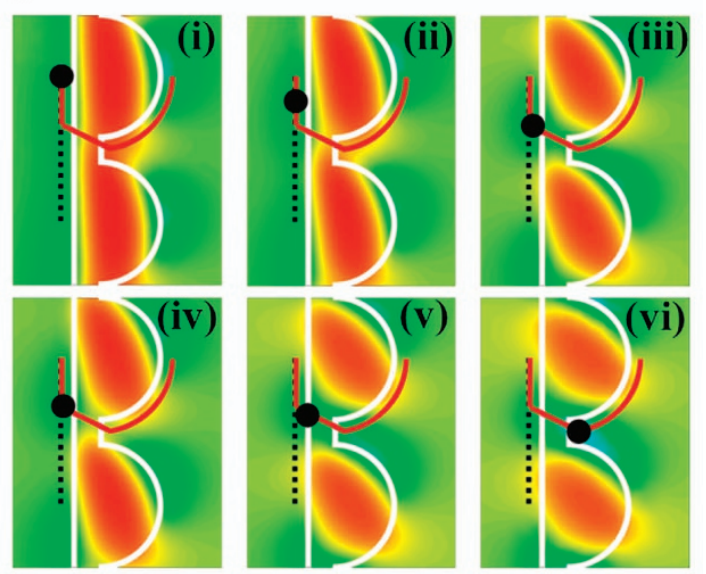

Figure 4 Magnetic potential energy of half-disk patterns in the magnetic spider web. (a) The minimum potential energy is plotted for a clockwise rotating field along patterns 1 and 2 in T-junction. The energy minima allow the particle to cross the T-junction and move toward the linear forward direction instead of going to flat edge. The pattern 1 (red line) has the minimum energy than the pattern 2 (black dotted line). The inset shows the schema of the particle trajectory along patterns 1 and 2. (b) The minimum potential energy is plotted for a counterclockwise rotating field along the patterns (1) and (2). The minimum potential energy is lowered when the particle moves along pattern 2 instead of pattern 1 which preventing the crossing over a linear segment because of the presence of energy barrier at the junction point make obstacle for the particle movement (the inset shows the schematic of the particle trajectory along each path). (c) The minimum potential energy is plotted for a counterclockwise rotating field along the edge pattern of spiral. Inset shows the schematic of the edge of the spiral pattern (pattern 3). The lower energy minima of linear curve of pattern 3 than that of flat surface indicates that the particles cannot move on the flat surface toward the flat edge of pattern 2 and revolve around edge of the pattern 3 by applying counterclockwise rotating field. (d) The potential energy landscape of particles indicates the local energy minimums from $\theta=-60^{\circ}$ to $-50^{\circ}$ are revealed to across the narrow connection between patterns 2 and 3 (blue and red colors designate the energy minima and maxima, respectively).

Information,Supplementary Figure S3b). Here, the $\mathrm{x}$-component of the field induces the sensor signal, and is given as the following equation: ${ }^{38}$

$$
B_{\mathrm{x}}=\frac{\mu_{0}}{4 \pi} \frac{\chi_{V} V_{\text {particle }} H_{\text {app }}}{r^{3}}\left(2 \cos ^{2} \theta-\sin ^{2} \theta\right)
$$

with

$$
\theta=\cos ^{-1}\left(\frac{x}{\left(x^{2}+y^{2}+z^{2}\right)^{1 / 2}}\right), r=\left(x^{2}+y^{2}+z^{2}\right)^{1 / 2}
$$

where $\chi_{\mathrm{V}}$ is the volume susceptibility of the particle, $V_{\text {particle }}$ is volume and $r$ is distance from particle center. Here, the particles are superparamagnetic and the external field is much smaller than the saturation field, as shown in Supplementary Figure S3c. The calculated mean magnetic moment per particle is $6.4 \times 10^{-14} \mathrm{~A} \mathrm{~m}^{2}$, where the density of the particles is $1.4 \times 10^{3} \mathrm{~kg} \mathrm{~m}^{-3} .39$

It is noted that magnitude of the effective field on the sensor surface is always smaller than that of the applied field because the effective field is the vector sum of the applied and the stray fields. Figure $5 c$ shows the calculated field distribution Equation (2) with $z$ equal to sum of particle radius $(1.4 \mu \mathrm{m})$ and passivation layer of sensor $(700 \mathrm{~nm})$, where the minimum field is revealed $(-691.4 \mu \mathrm{T})$ near the particle center, and the maximum field $(139.6 \mu \mathrm{T})$ is revealed for outside of particle $(x= \pm 2.5 \mu \mathrm{m})$. The stray field distributions for paired two particles in $x$-, $y$ - and $z$-axis are different (Supplementary Figure S4) and their minimum values are 554, 797 and $746 \mu \mathrm{T}$, respectively.

When the neighboring particle number increases on two-dimensional sensor surface, the total field on the sensor could 

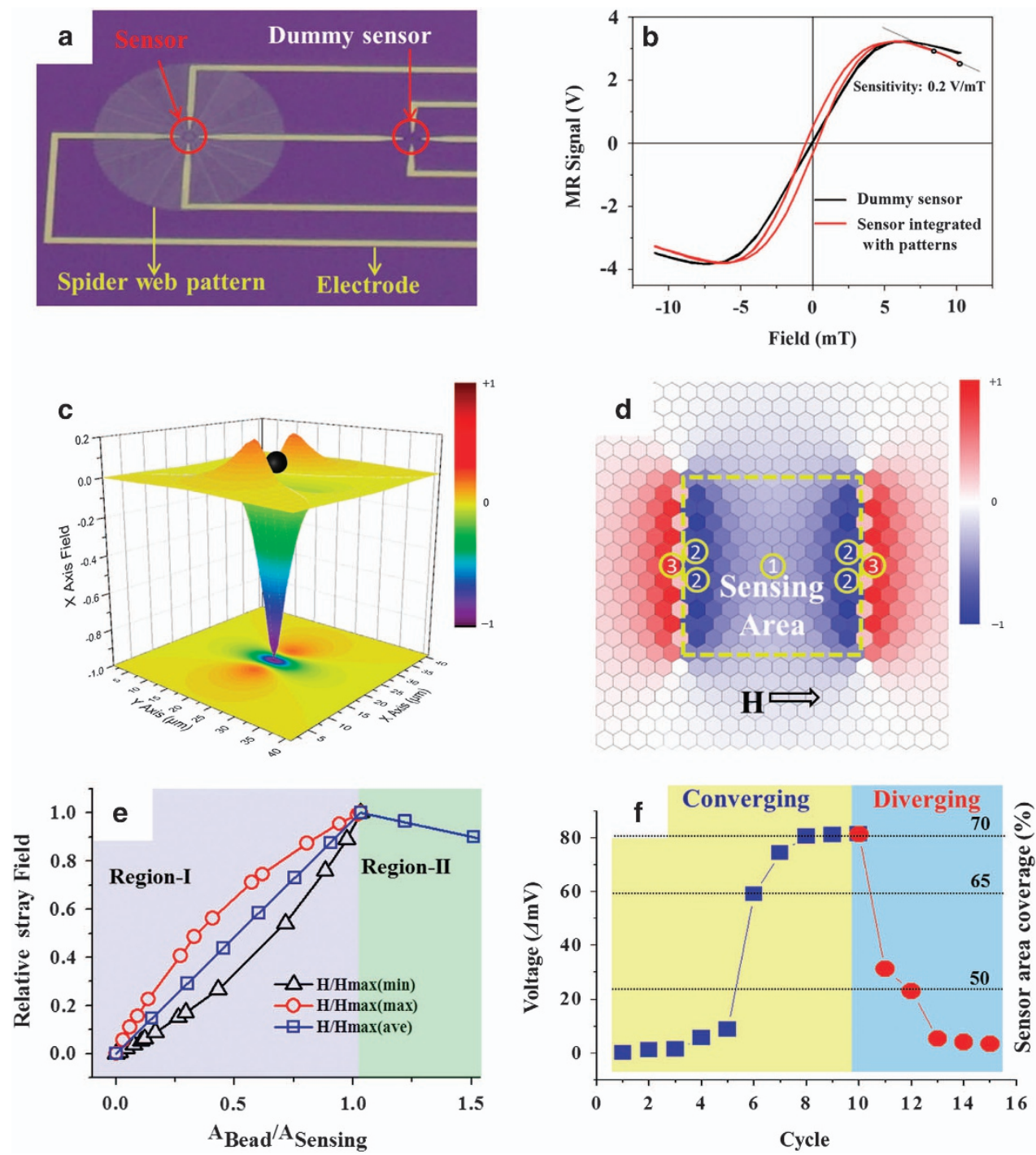

Figure 5 Simultaneous manipulation and monitoring of magnetic particle. (a) A photo of the magnetic spider web chip integrated with the magnetoresistive (MR) sensor at the web center and the dummy sensor fabricated in the same batch. (b) The MR signal profiles for dummy and active sensors integrated with the spider web patterns. (c) Calculated stray field distribution of single particle on sensor surface; color bar is normalized by $-691.4 \mu \mathrm{T}$. (d) Landscape of effective stray field depending on the particle location with respect to sensing area of the sensor, $-1.130 \mu \mathrm{T}$ near center (light blue), marked as (1), $-3.487 \mu \mathrm{T}$ near edge (dark blue), marked as (2), and positive $3.069 \mu \mathrm{T}$, marked as (3) (red color); color bar is normalized by $-3.487 \mu \mathrm{T}$. (e) Relative of stray field variation as a function of increasing particle coverage area (blue line: Hmax(ave), average stray field; red line: Hmax(max), descending order stray field inside sensing area; black line: Hmax(min), ascending order of stray field inside sensing area). (f) The MR signal during particle converging and diverging at the sensor surface, where the MR signal variation becomes saturated and desaturated after only a few rotating field cycles. The percentage of coverage of the sensor surface that resulted with particle accumulation based on rotating field cycles is presented in (f).

be the vector sum of the field distribution of individual particles. Figure $5 \mathrm{~d}$ shows the landscape of summing effective field, $\overline{B_{\mathrm{x}}}=\int_{A_{\text {sensor }}} B_{\mathrm{x}} d A / A_{\text {sensor }}$, depending on the particle location for hexagon close packing structure with respect to two-dimensional sensing area, where $30 \times 30 \mu \mathrm{m}^{2}$ sensing area was assumed. As a whole, the particles located inside the sensing area induce the negative field (blue color), but the particles located outside the sensing area cause the positive field (red color). In addition, single particles cause different effective fields, even though they are located inside the sensing area, that is, $\sim-1.130 \mu \mathrm{T}$ near center (light blue) and $\sim-3.487 \mu \mathrm{T}$ near edge (dark blue).

The effective field on sensor increases with the particle coverage, $A_{\text {particle }} / A_{\text {sensing, up to }} 1$ if it is just sum of individual stray field, as shown in Figure 5e, where the increasing rate changes depending on the particle accumulating location (region I). The increasing rate of effective field for accumulated particles from the edge is larger, marked as (1) than that for particles accumulating from the center, marked as (2) (Figure 5d). However, the particles located outside the sensing area induce positive field, and thus further increasing particles outside the sensor cause the decrement in the total stray field (region II). When the coverage of accumulated particle $A_{\text {particle }} / A_{\text {sensing }}$ was approximated to be ' 1 ', the effective field was estimated to be $\sim 0.34 \mathrm{mT}$.

The MR sensor signal from the electrodes in the $y$-direction was measured as the magnetic field was swept in the $y$-direction, where a fixed current of $\mathrm{I}=1 \mathrm{~mA}$ was in the same direction as the exchange coupling field, that is, through the electrodes in the $x$-direction. The MR signal profile in the sensor was amplified by 1000 times. 
Thus, the MR signal near $+7.96 \mathrm{kA} \mathrm{m}^{-1}$ regime (signal decreases with the field) increases as the stray field increases. However, the signal near $-7.96 \mathrm{kA} \mathrm{m}^{-1}$ (signal increases with the field) decreases as the stray field increases, and the opposite occurs in a diverging process. The full MR signals were recorded under an AC magnetic field applied along the $y$-axis after each cycle of magnetic field rotation (Supplementary Figure S5a), and the signal variation was obtained by averaging $\Delta V_{1}$ and $\Delta V_{2}$ for converging and diverging processes.

The measured MR variations $\Delta V$ during the convergence step are depicted in the Supplementary Figures (Supplementary Figures S5b and c) after 0, 2, 4 and 6 cycles of a counterclockwise rotating field and a diverging step. The measured MR signal is depicted in Supplementary Figures S5d and e (Supplementary Information) after 0,2 and 4 cycles of a counterclockwise rotating field. The time variation (cycle rotation) of the MR signal is shown in Figure $5 \mathrm{f}$. Based on measured MR signal $(81.25 \mathrm{mV})$ and the sensor sensitivity $\left(0.2 \mathrm{~V} \mathrm{mT}^{-1}\right)$, the effective field is calculated to be nearly $0.4 \mathrm{mT}$, and this is good agreement with the theoretical approximation $(0.34 \mathrm{mT})$. The percentage of coverage of the sensor surface with the resulting accumulated number of particles from each measurement points was estimated by ImageJ software (US National Institutes of Health, Bethesda, MD, USA, see Supplementary Information) and marked in Figure 5f. Here, the MR signal maxima were obtained at the coverage of $70 \%$, because the pattern area overlapped on the sensor surface cannot accommodate any particle (Supplementary Figure S6). The asymmetry in the time dependence of the convergence and divergence of the MR sensor signals reflects the state of initial particle dispersion and its area density on the spider web. In this magnetic spider technique, few numbers of particles can be detected by integrated MR sensor. Thus, this spider web pattern demonstrates the principle underlying the controlled transportation of biomolecule carriers even in low density that can improve the biosensor sensitivity and efficiency.

\section{CONCLUSION}

In summary, a novel technique was developed for the simultaneous manipulation and monitoring of biomolecules using a magnetic spider web consisting of several linear radii and spiral tracks. This design was successfully applied to the concentric collection of protein-coated superparamagnetic carrier particles at the symmetric spider web center. The integrated PHR sensor at the spider web center allows the detection of labeled particles. The voltage variation increases with the number of collected particles on the sensor and returns to the original value as the particles move away from the sensor surface. This novel platform could possibly open a new biological assay system for future diagnostic devices that overcomes potential issues facing existing nano/micro-biosensors, that is, the limitations regarding detecting particles that are distant from the sensor surface, and allows us to accurately manipulate and detect bioanalytes.

\section{CONFLICT OF INTEREST}

The authors declare no conflict of interest.

\section{ACKNOWLEDGEMENTS}

This research was supported by the BioNano Health Guard Research Center (Grant Number H-GUARD_2013M3A6B2078959) and National Research Foundation (No 2010-0027963) funded by the Korean government (MISP).
1 Arlett, J. L., Myers, E. B. \& Roukes, M. L. Comparative advantages of mechanical biosensors. Nat. Nanotechnol. 6, 203-215 (2011).

2 Sheehan, P. E. \& Whitman, L. J. Detection limits for nanoscale biosensors. Nano Lett. 5, 803-807 (2005).

3 Takeda, S., Wegmann, S., Cho, H., DeVos, S. L., Commins, C., Roe, A. D., Nicholls, S. B., Carlson, G. A., Pitstick, R., Nobuhara, C. K., Costantino, I., Frosch, M. P., Müller, D. J., Irimia, D. \& Hyman, B. T. Neuronal uptake and propagation of a rare phosphorylated high-molecular-weight tau derived from Alzheimer's disease brain. Nat. Commun. 6, 8490 (2015).

4 Prindle, A., Samayoa, P., Razinkov, I., Danino, T., Tsimring, L. S. \& Hasty, J. A sensing array of radically coupled genetic 'biopixels'. Nature 481, 39-44 (2012).

5 Akin, D., Li, H. \& Bashir, R. Real-time virus trapping and fluorescent imaging in microfluidic devices. Nano Lett. 4, 257-259 (2004).

6 Ko, H., Lee, J., Kim, Y., Lee, B., Jung, C. H., Choi, J. H., Kwon, O. S. \& Shin, K. Active digital microfluidic paper chips with inkjet-printed patterned electrodes. Adv. Mater. 26, 2335-2340 (2014).

7 Kim, K. W., Reddy, V., Torati, S. R., Hu, X. H., Sandhu, A. \& Kim, C. G. On-chip magnetometer for characterization of superparamagnetic nanoparticles. Lab Chip 15, 696-703 (2015).

8 Waggoner, P. S., Varshney, M. \& Craighead, H. G. Detection of prostate specific antigen with nanomechanical resonators. Lab Chip 9, 3095-3099 (2009).

9 Kurosawa, S., Nakamura, M., Park, J. W., Aizawa, H., Yamada, K. \& Hirata, M. Evaluation of a high-affinity QCM immunosensor using antibody fragmentation and 2 methacryloyloxyethyl phosphorylcholine (MPC) polymer. Biosens. Bioelectron. 20, 1134-1139 (2004).

10 Weizmann, Y., Patolsky, F. \& Willner, I. Amplified detection of DNA and analysis of single-base mismatches by the catalyzed deposition of gold on Au-nanoparticles. Analyst 126, 1502-1504 (2001).

11 Srinivasan, B., Li, Y., Jing, Y., Xu, Y., Yao, X., Xing, C. \& Wang, J. P. A detection system based on giant magnetoresistive sensors and high-moment magnetic nanoparticles demonstrates zeptomole sensitivity: potential for personalized medicine. Angew. Chem. Int. 48, 2764-2767 (2009).

12 Gaster, R. S., Hall, D. A., Nielsen, C. H., Osterfeld, S. J., Yu, H., Mach, K. E., Wilson, R. J., Murmann, B., Liao, J. C., Gambhir, S. S. \& Wang, S. X. Matrix-insensitive protein assays push the limits of biosensors in medicine. Nat. Med. 15, 1327-1332 (2009)

13 Squires, T. M., Messinger, R. J. \& Manalis, S. R. Making it stick: convection, reaction and diffusion in surface-based biosensors. Nat. Biotechnol. 26, 417-426 (2008).

14 Bejhed, R. S., Tian, B., Eriksson, K., Brucas, R., Oscarsson, S., Strömberg, M., Svedlindh, P. \& Gunnarsson, K. Magnetophoretic transport line system for rapid on-chip Attomole protein detection. Langmuir 31, 10296-10302 (2015).

15 Kelley, S. O., Mirkin, C. A., Walt, D. R., Ismagilov, R. F., Toner, M. \& Sargent, E. H. Advancing the speed, sensitivity and accuracy of biomolecular detection using multilength-scale engineering. Nat. Nanotechnol. 9, 969-980 (2014).

16 Baselt, D. R., Lee, G. U., Natesan, M., Metzger, S. W., Sheehan, P. E. \& Colton, R. J. A biosensor based on magnetoresistance technology. Biosens. Bioelectron. 13, 731-739 (1998).

17 Sinha, B., Ramulu, T. S., Kim, K. W., Venu, R., Lee, J. J. \& Kim, C. G Planar Hall magnetoresistive aptasensor for thrombin detection. Biosens. Bioelectron. 59, 140-144 (2014).

18 Brzeska, M., Panhorst, M., Kamp, P. B., Schotter, J., Reiss, G., Pühler, A., Becker, A. \& Brückl, H. Detection and manipulation of biomolecules by magnetic carriers. J. Biotechnol. 112, 25-33 (2004).

19 Hung, T. Q., Terki, F., Kamara, S., Kim, K., Charar, S. \& Kim, C. G. Planar Hall ring sensor for ultra-low magnetic moment sensing. J. Appl. Phys. 117, 154505 (2015).

20 Janssen, X. J., van IJzendoorn, L. J. \& Prins, M. W. On-chip manipulation and detection of magnetic particles for functional biosensors. J. Biosens. Bioelectron. 23, 833-838 (2008).

21 Donolato, M., Torti, A., Kostesha, N., Deryabina, M., Sogne, E., Vavassori, P., Hansen, M. F. \& Bertacco, R. Magnetic domain wall conduits for single cell applications. Lab Chip 11, 2976-2983 (2011).

22 Jokilaakso, N., Salm, E., Chen, A., Millet, L., Guevara, C. D., Dorvel, B., Reddy, B. Jr, Karlstrom, A. E., Chen, Y., Ji, H., Chen, Y., Sooryakumar, R. \& Bashir, R. Ultra-localized single cell electroporation using silicon nanowires. Lab Chip 13, 336-339 (2013).

$23 \mathrm{Hu}$, X., Goudu, S. R., Torati, S. R., Lim, B., Kim, K. \& Kim, C. An on-chip micromagnet frictionometer based on magnetically driven colloids for nano-bio interfaces. Lab Chip 16, 3485-3492 (2016).

24 Yellen, B. B., Erb, R. M., Son, H. S., Hewlin, R. Jr, Shang, H. \& Lee, G. U. Traveling wave magnetophoresis for high resolution chip based separations. Lab Chip 7, 1681-1688 (2007).

$25 \mathrm{Li}$, P., Mahmood, A. \& Lee, G. U. Flow-Enhanced nonlinear magnetophoresis for highresolution bioseparation. Langmuir 27, 6496-6503 (2011).

26 Lim, B., Reddy, V., Hu, X., Kim, K., Jadhav, M., Abedini-Nassab, R., Noh, Y. W., Lim, Y. T., Yellen, B. B. \& Kim, C. G. Magnetophoretic circuits for digital control of single particles and cells. Nat. Commun. 5, 1 (2014).

27 Pushkarsky, I., Tseng, P., Murray, C. \& Carlo, D. D. Research highlights: microfluidics and magnets. Lab Chip 14, 2882-2886 (2014).

28 Abedini-Nassab, R., Joh, D. Y., Triggiano, M. A., Baker, C., Chilkoti, A., Murdoch, D. M. \& Yellen, B. B. Magnetophoretic conductors and diodes in a $3 d$ magnetic field. Adv. Funct. Mater. 26, 426-4034 (2016). 
29 Gunnarsson, K., Roy, P. E., Felton, S., Pihl, J., Svedlindh, P., Berner, S., Lidbaum, H. \& Oscarsson, S. Programmable motion and separation of single magnetic particles on patterned magnetic surfaces. Adv Mater. 17, 1730-1734 (2005).

30 Conroy, R. S., Zabow, G., Moreland, J. \& Koretsky A., P. Controlled transport of magnetic particles using soft magnetic patterns. Appl. Phys. Lett. 93, 203901 (2008).

31 Vieira, G., Henighan, T., Chen, A., Hauser, A. J., Yang, F. Y., Chalmers, J. J. \& Sooryakumar, R. Magnetic wire traps and programmable manipulation of biological cells. Phys. Rev. Lett. 103, 128101 (2009).

32 Hu, X., Torati, S. R., Shawl, A. I., Lim, B., Kim, K. \& Kim, C. Micromagnet conductors for high-resolution separation of magnetically driven particles and cells by multifrequencies. IEEE Magn. Lett. 7, 1508004 (2016).

33 Donolato, M., Vavassori, P., Gobbi, M., Deryabina, M., Hansen, M. F., Metlushko, V., Ilic, B., Cantoni, M., Petti, D., Brivio, S. \& Bertacco, R. On-chip manipulation of proteincoated magnetic beads via domain-wall conduits. Adv. Mater. 22, 2706-2710 (2010).

34 Hung, T. Q., Terki, F., Kamara, S., Dehbaoui, M., Charar, S., Sinha, B., Kim, C. G., Gandit, P., Guralskiy, I. A., Molnar, G., Salmon, L., Shepherd, H. J. \& Bousseksou, A. Room temperature magnetic detection of spin switching in nanosized spin-crossover materials. Angew. Chem. Int. Ed. 52, 1185-1188 (2013).

$35 \mathrm{Kim}$, C. Seo, J. H. \& Parvatheeswara Rao, B. Depth sensitive exchange coupled spin structure in NiFe/FeMn bilayer. J. Appl. Phys. 102, 113904 (2007).

36 Lim, B., Vavassori, P., Sooryakumar, R. \& Kim, C Nano/micro-scale magnetophoretic devices for biomedical applications. J. Phys. D Appl. Phys. 50, 033002 (2017).
$37 \mathrm{Kralj}$, S. \& Makovec, D. Magnetic assembly of superparamagnetic iron oxide nanoparticle clusters into nanochains and nanobundles. ACS Nano 9, 9700-9707 (2015).

38 O'handley, R. C. (ed.) Modern Magnetic Materials, Principles and Applications. Chapter 2, 54 (John Wiley \& Sons Inc., New York, USA, 2000).

$39 \mathrm{Hu}$, X., Lim, B., Jeong, I., Sandhu, A. \& Kim, C Optimization of pathway pattern size for programmable biomolecule actuation. IEEE T. Magn. 49, 408-413 (2013).

\section{(c) (i)}

This work is licensed under a Creative Commons Attribution 4.0 International License. The images or other third party material in this article are included in the article's Creative Commons license, unless indicated otherwise in the credit line; if the material is not included under the Creative Commons license, users will need to obtain permission from the license holder to reproduce the material. To view a copy of this license, visit http:// creativecommons.org/licenses/by/4.0/

(C) The Author(s) 2017

Supplementary Information accompanies the paper on the NPG Asia Materials website (http://www.nature.com/am) 